\title{
Chagas disease and triatomine biology (Heteroptera, Triatominae), with emphasis on aspects of spermatogenesis
}

\author{
R. Silistino-Souza
}

2011. Universidade Estadual Paulista “Júlio de Mesquita Filho", São José do Rio Preto, SP, Brasil. Ph.D. thesis. Orienting Prof.: Maria Tercília Vilela de Azeredo Oliveira DOI http://dx.doi.org/10.4238/vol10-3ta039

A century after the discovery of Chagas disease, it is still one of the most important parasitic diseases affecting humans. The subfamily Triatominae is important in medical health, because these insects are vectors of Trypanosoma cruzi, the etiologic agent of Chagas disease. These insects are also of important cytological relevance because they have particular cell characteristics, such as persistence of nucleolar material in spermatogenesis. The germ cells of the animal kingdom have chromatoid bodies (CBs) in their cytoplasm that can originate from nucleolar material that is fragmented in the early stages of spermatogenesis and plays an important role in cellular communication between the spermatids during spermiogenesis. Currently, there are few studies on the function and formation of the $\mathrm{CB}$ in nucleologenesis, especially with emphasis on the ultrastructure of the cells involved in spermatogenesis of insects. Considering the importance of knowledge about the triatomine fauna, we conducted a study of the biogeography and reports of these insects and a survey of patients with Chagas disease in the northwestern region of São Paulo State. Data collected from 1995 to 2009 indicated 700 individuals with Chagas disease, demonstrating a range of 0 to 40 years, which shows that the disease may be active in this region. Moreover, of the 1150 patients treated for cardiomyopathy, 44\% were chagasic. Regarding the triatomines noted and captured in the period from 2004 to 2009, the species were Triatoma sordida and Rhodnius neglectus, with T. sordida being the most abundant. In addition, some triatomines were infected by $T$. cruzi in various developmental stages. We also analyzed the nucleolar cycle and fibrillarin nucleolar protein expression in CB of spermatogenic cells of T. infestans and T. sordida, using histological, ultrastructural and immunocytochemical techniques. The results revealed fibrillarin nucleolar protein expression in the nucleus and in some cytoplasmic spots of germ cells during spermatogenesis in triatomines. These data suggest that fibrillarin could be a constituent of $\mathrm{CB}$, which was most likely derived from nucleolar fragmentation. This is the first time that fibrillarin protein expression has been shown in CB during spermatogenesis progression in triatomines. Knowledge about the biology of triatomines was deepened in this study and, in particular, the structural and ultrastructural aspects of spermatogenesis in triatomines. This study showed that the disease may be active in the northwestern region of São Paulo and expanded our knowledge of the biology of triatomines, the main vectors of Chagas disease.

Key words: Chagas disease; Chromatoid body; Triatoma infestans; Triatoma sordida; Rhodnius neglectus 